\title{
EFFECT OF GALLIUM FOCUSED ION BEAM IRRADIATION ON PROPERTIES OF $\mathrm{YBa}_{2} \mathrm{Cu}_{3} \mathrm{O}_{x} / \mathrm{La}_{0.67} \mathrm{Sr}_{0.33} \mathrm{MnO}_{3}$ HETEROSTRUCTURES
}

\author{
Vladimír Štrbík - Štefan Beňačka — Štefan Gaži — Vasilij Šmatko \\ — Štefan Chromik - Agáta Dujavová — Ivo Vávra
}

\begin{abstract}
We present initial investigation of the superconductor-ferromagnet-superconductor (SFS) heterostructures of nanometer dimensions prepared by the gallium focused ion beam (FIB) technology. The SFS heterostructures were realized on the basis of high-Tc superconducting $\mathrm{YBa}_{2} \mathrm{Cu}_{3} \mathrm{O}_{x}$ and ferromagnetic $\mathrm{La}_{0.67} \mathrm{Sr}_{0.33} \mathrm{MnO}_{3}$ thin films. SFS weak link junctions require dimensions of the weak link connection in the range of nanometer size realizable by FIB patterning. On the other side the gallium focused ion beam might bring about unacceptable degradation of the superconducting as well as ferromagnetic thin film properties. The presented results show that FIB offers a suitable procedure for realization of nanometer size devices but some degradation of the ferromagnetic and superconducting properties was observed. Solution of this problem will be achieved in the next stage of our investigations.
\end{abstract}

K e y w ords: superconductor-manganite junctions, proximity effect, magnetic domain wall

\section{INTRODUCTION}

Superconductor-ferromagnet (SF) bilayer and SFS trilayer heterostructures, in the form of weak links or Josephson junctions, are very attractive objects for the study of mutual interplay between superconductivity and ferromagnetism [1]. In addition, their potential applications are very promising in cryoelectronic or cryospintronic circuits [2] (eg, qubits, 0-, pi-junctions, spin valves, etc). However, utilization of high-Tc superconductors in this area was not successful until now. In the case of the high-Tc cuprate superconductor $\mathrm{YBa}_{2} \mathrm{Cu}_{3} \mathrm{O}_{x}$ (YBCO), as convenient ferromagnetic $(\mathrm{F})$ materials are manganites, eg $\mathrm{La}_{0.67} \mathrm{Sr}_{0.33} \mathrm{MnO}_{3}$ (LSMO) - ferromagnetic perovskite half metal, which may be totally spin polarized in one spin direction. YBCO/LSMO heterostructures, prepared on single crystal $\mathrm{MgO}$ or $\mathrm{SrTiO}_{3}$ substrate, are able to create high quality thin films and SF interfaces necessary for the study of the physical properties. In addition, present advanced technologies allow realization of structures of nanometer dimensions which open new possibilities for preparation of high-Tc superconducting weak links with properties similar to low-Tc Josephson junctions, however operating at much higher temperatures. In this paper we describe the first stage of preparation and the properties of $\mathrm{YBCO} / \mathrm{LSMO} / \mathrm{YBCO}$ nanometer heterostructures, using the technology of gallium focused ion beam (FIB) patterning, to find out whether this technology is a suitable tool for realization of superconductor weak link structures.

Generally, electron transport through the interface between the superconductor $(\mathrm{S})$ and normal $(\mathrm{N})$ or ferro- magnetic $(\mathrm{F})$ metal in close proximity with $\mathrm{S}$, is mediated by Andreev reflection [3], the process in which an electron with energy smaller than the energy gap of $\mathrm{S}$ is reflected as a hole preserving in $\mathrm{N}(\mathrm{F})$ phase coherence of Cooper pairs $(\mathrm{CP})$ over some distance from the interface. There are essential differences between the proximity effect in $\mathrm{SN}$ and SF structures. In SN connection the penetration depth of singlet spin $(\uparrow \downarrow)$ CP into the $\mathrm{N}$ metal is determined by the coherence length $\xi_{N}=\left(\hbar D_{N} / 2 \pi k_{B} T\right)^{1 / 2}$, where $D_{N}$ is the diffusion coefficient in N, T is temperature, and $\hbar$ and $k_{B}$ are the Planck and Boltzmann constants. At very low temperatures $\xi_{N}$ can reach several micrometers. In the case of a SF bilayer the penetration depth of singlet spin CP into the ferromagnet is much shorter $\xi_{F}=\left(\hbar D_{F} / 2 \pi E_{e x}\right)^{1 / 2}$ provided that the magnetic exchange energy $E_{e x} \gg k_{B} T$ is rather large, which is fulfilled in the case of perovskite (half metal) materials. Fortunately, in addition to the short coherence length $\xi_{F}(\sim 1-2 \mathrm{~nm})$ there was discovered a long range proximity effect (LRPE) in FS structures, in case inhomogeneous magnetization in the vicinity of SF interface is present [4]. It was also shown [5] that such inhomogeneity (eg, domain wall, spin active interface) generates a triplet spin $\mathrm{CP}$, with amplitude comparable to the singlet one, containing phase correlations between electrons with the same spin projections $(\uparrow \uparrow)$, on the coherence length $\xi_{F L}$. The penetration depth in the case of LRPE should be of the order $\xi_{N}$ as in the case of singlet spin CP into a normal metal. Large attention is therefore dedicated to the investigation of Josephson effects in cuprate SFS struc-

* Institute of Electrical Engineering, SAS, Dúbravská cesta 9, 84104 Bratislava, Slovakia, elekstrb@savba.sk 


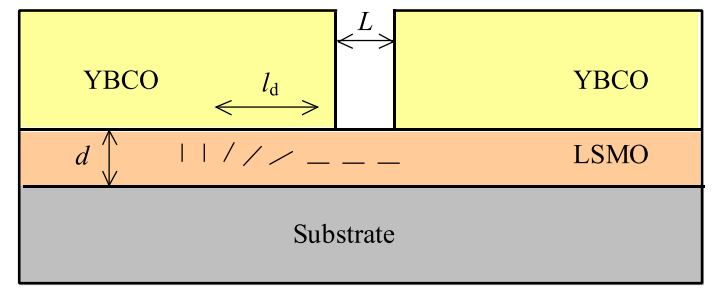

Fig. 1. Lateral geometry of the SFS microstrip structure. Magnetic inhomogeneity in LSMO of thickness $d$ is intended to be realized by domain wall of width $l_{d}$. The two YBCO electrodes are separated in length $L$ as shown

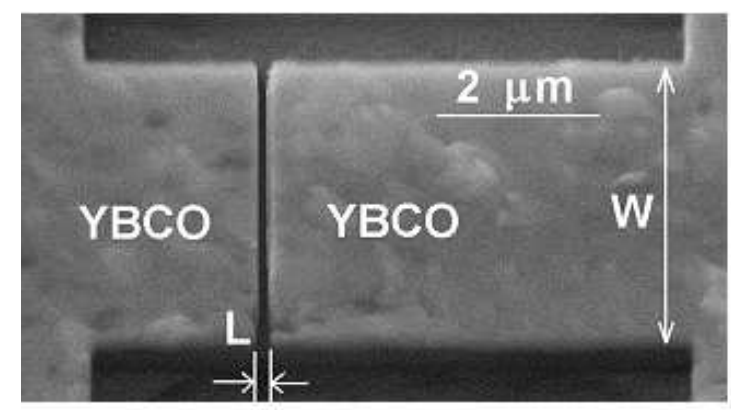

Fig. 2. The YBCO/LSMO microstrip $\left(7 \times 4 \mu \mathrm{m}^{2}\right)$ with a gap in YBCO thin film (length $L \approx 70-80 \mathrm{~nm}$ ) created by FIB etching. This SFS structure represents YBCO/LSMO/YBCO junction of lateral geometry

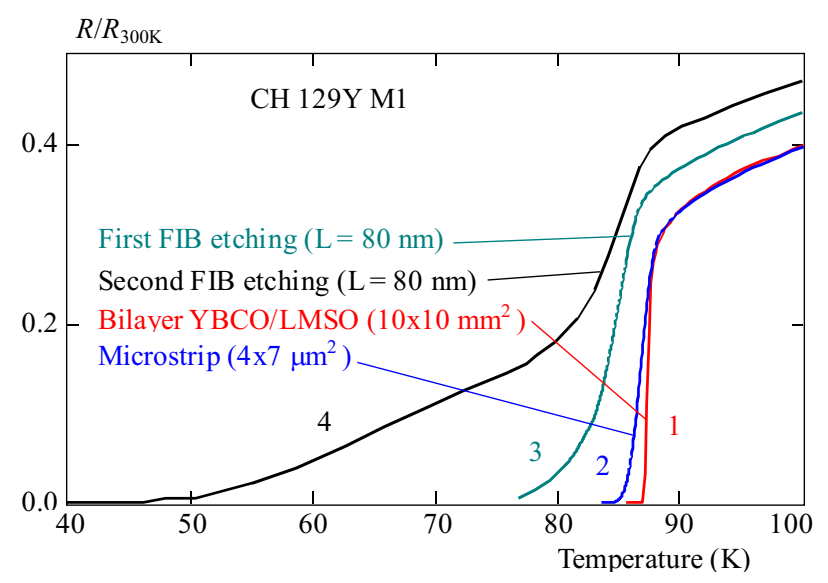

Fig. 3. The normalized R-T dependences of four sequences of SFS structure preparation: 1- as prepared bilayer film, 2- bilayer microstrip $\left(7 \times 4 \mu \mathrm{m}^{2}\right)$, 3- after the first FIB etching of the gap in YBCO, and 4- after the second FIB etching of the gap

tures due to possible larger variety of Josephson junction properties realized on these materials combination [6].

Realization of high quality high-Tc SF or SFS structures manifesting LRPE is a complicated task. The magnetic inhomogeneity, in the so-called series geometry, must be localized immediately at the SF interface, which is experimentally extremely difficult, otherwise the triplet current amplitude is negligibly small. Recently, it was analysed [7] that, in comparison with the serial geometry, the amplitude of the triplet current component may be in the so- called lateral geometry (Fig. 1) enhanced by factor $l_{d} / d$, where $l_{d}$ is the domain wall width and $d$ is the thickness of $\mathrm{F}$ thin film. The domain wall width [8] $l_{d}=\pi(A / K)^{1 / 2}$, where $A$ is the exchange energy acting to keep spins parallel and $K$ is the energy of magnetization anisotropy. Competition of these energies results in a finite width of the domain wall. Recently, the values of $A$ and $K$ were extracted for LSMO from $\mathrm{LSMO} / \mathrm{SrRuO}_{3}$ bilayers and superlattices [9] as $A=5 \times 10^{-12} \mathrm{~J} / \mathrm{m}$ and $K=-5 \times 10^{3} \mathrm{~J} / \mathrm{m}^{3}$, thus the domain wall width may receive values in the range of $100 \mathrm{~nm}$. In reality the values of $A$ and $K$ depend on composition, temperature, stress in the $\mathrm{F}$ film, consequently, for $l_{d} / d \gg 1$ the high quality and small thickness (10-20 nm) of the ferromagnetic film is required to bring LRPE coherence length $\xi_{F L}$ closer to $\xi_{N}$.

In the case of lateral geometry the LRPE should be independent of the domain wall localization [7], in the immediate vicinity of SF interface, what enables considerable simplification of technological conditions. In the ongoing experimental investigation we will henceforth verify these analyzes.

\section{EXPERIMENTAL}

The dc magnetron sputtering was used for in situ growing the bilayer heterostructure $\mathrm{YBa}_{2} \mathrm{Cu}_{3} \mathrm{O}_{x}$ (YBCO) and $\mathrm{La}_{0.67} \mathrm{Sr}_{0.33} \mathrm{MnO}_{3}$ (LSMO) on single crystal $\mathrm{MgO}$ (100) substrate. The single LSMO thin films showed transition to the metallic ferromagnetic state at about $200 \mathrm{~K}$ and their resistivity at liquid nitrogen temperature was $\rho \approx 10^{-3} \Omega \mathrm{cm},[10]$. The thickness of LSMO layers was in the range $20-50 \mathrm{~nm}$ where the LSMO thin film should be ferromagnetic [11]. The LSMO crystallizes as pseudocubic perovskite, it has a fully spinpolarized conduction band and bulk material exhibits ferromagnetic transition around room temperature. The YBCO films were deposited applying high pressure onaxis dc magnetron sputtering carried out at oxygen pressure $300 \mathrm{~Pa}$, substrate temperature $T_{s}=810^{\circ} \mathrm{C}$, and dc power $200 \mathrm{~W}$, with a deposition rate of $1 \mathrm{~nm} / \mathrm{min}$ [12], [13]. The thickness of the YBCO superconducting films was about $150 \mathrm{~nm}$.

Patterning of the basic bilayer structures for four point measurement of the transport properties was carried out by optical photolithography and wet $\left(1 \% \mathrm{H}_{3} \mathrm{PO}_{4}\right)$ or $\mathrm{Ar}$ ion beam etching $\left(300 \mathrm{eV}, 20 \mathrm{~mA} / \mathrm{cm}^{2}\right)$, with substrate cooled to about minus $20^{\circ} \mathrm{C}$. Subsequently $\mathrm{Ga}^{+3}$ focused ion beam patterning was applied (Quanta 3D 200i) to receive the convenient lateral geometry (Fig. 1).

\section{RESULTS AND DISCUSSION}

Zero resistance critical temperature $T_{C 0}$ of single $c$ axis oriented YBCO films, deposited directly on single crystal $\mathrm{MgO}$ substrate, was typically somewhat below $90 \mathrm{~K}$, the critical current density at $77 \mathrm{~K} j_{C}(77 \mathrm{~K}) \approx$ 


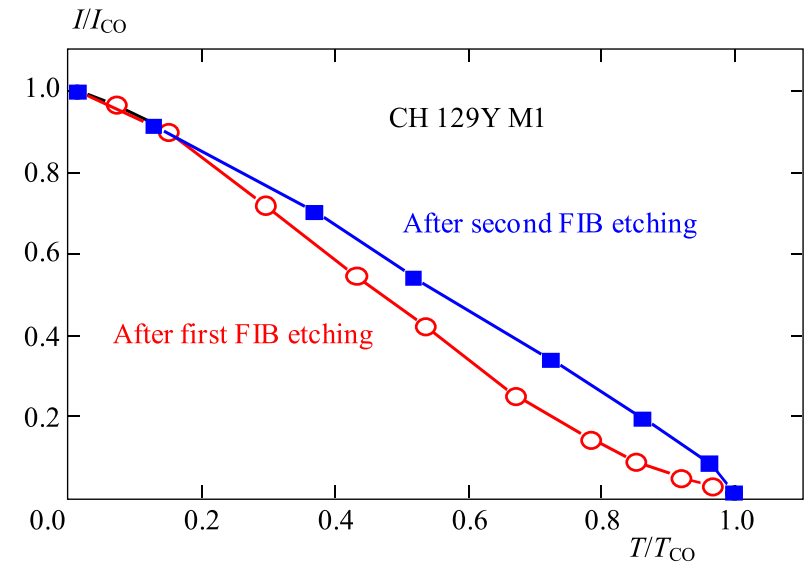

Fig. 4. Normalized dependences of the critical current $v s$ temperature for $\mathrm{YBCO} / \mathrm{LSMO} / \mathrm{YBCO}$ structure after the first (open circles) and the second (filled squares) FIB etching of the gap in YBCO

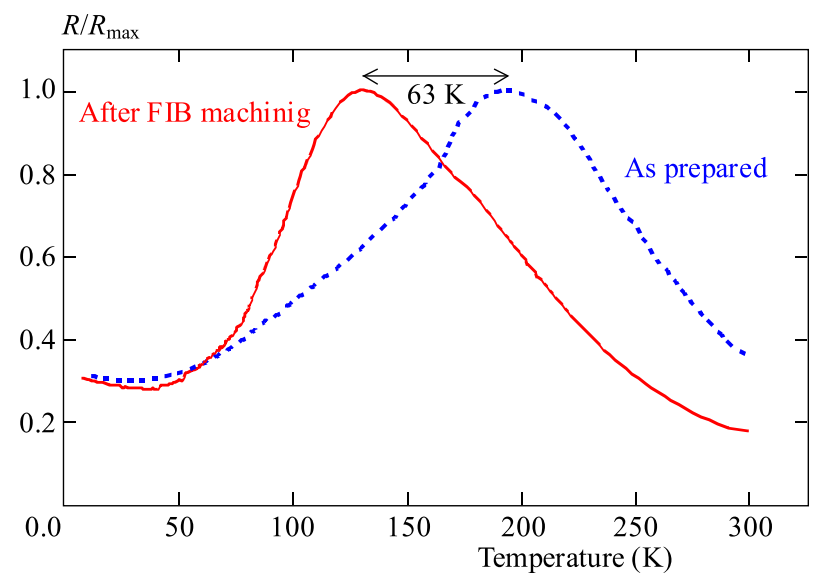

Fig. 5. $R-T$ dependences of SFS structure after removing the YBCO film in the gap (full line) and LSMO film not influenced by FIB irradiation (dashed line)

$3 \times 10^{6} \mathrm{~A} / \mathrm{cm}^{2}$ and the FWHM for the rocking curves of the (005) YBCO peak of $0.2^{\circ}$. A small decrease of $T_{C 0}$ and $j_{C}(77 \mathrm{~K})$ is due to the lattice mismatch between YBCO and $\mathrm{MgO}$. Zero resistance critical temperature of the YBCO/LSMO bilayers was above $80 \mathrm{~K}$, in the presented sample $T_{C 0}=87.5 \mathrm{~K}$ (Fig. 3, curve 1 ). In Fig. 2 we show SEM picture of YBCO/LSMO microstrip $\left(4 \times 7 \mu \mathrm{m}^{2}\right)$. The YBCO/LSMO bilayer was removed outside the microstrip by Ar ion beam. Subsequently the sample was transferred in Quanta 3D 200i and the gap of length $L$ in the YBCO film was realized by FIB etching. In addition the FIB etching was used for smoothing the microstrip edges.

The corresponding R-T dependence of the YBCO/LSMO microstrip is schown in Fig. 3, curve 2, at which a small decrease of $T_{C 0}$ to $85 \mathrm{~K}$ was observed due to the gallium ion irradiation. In the next step it was crucial to remove YBCO (without removing the LSMO thin film) as a narrow lateral gap of length $L$, to realize lateral geometry of the SFS structure. This is the most critical step of sample preparation because during the sample irradia- tion adjusted on the $30 \mathrm{keV} \mathrm{Ga}^{+3}$ ions the properties of superconductor and manganite films can be influenced.

After the first run of FIB etching (current $10 \mathrm{pA}$ and time duration of the procedure $7 \mathrm{~min}$ ) we obtained SFS weak link with relatively strong coupling of the two parts of YBCO. Zero resistance critical temperature $T_{C 0}$ decreased to $77 \mathrm{~K}$ (Fig. 3, curve 3). In the next run of FIB etching (current $10 \mathrm{pA}$ and time duration of the procedure $3 \mathrm{~min}$ ) we further weakened the SFS weak link coupling of the two YBCO parts, $T_{C 0}$ decreased to $43 \mathrm{~K}$, whereas the resistance of SFS structure increased by about $50 \%$.

The weakened coupling between both YBCO parts in lateral geometry can be documented by the temperature dependence of the critical current through the gap of length $L$. Extrapolated zero temperature value of $I_{C 0}$, after the first FIB etching of the gap, was $3 \mathrm{~mA}$ and corresponding temperature dependence of the critical current is shown in Fig. 4 by the open circle curve. The extrapolated zero temperature value of $I_{C 0}$, after the second FIB etching of the gap, reaches $0.3 \mathrm{~mA}$ and its temperature dependence is in Fig. 4 depicted by the curve with filled squares. The two $I_{C}-T$ dependences indicate a frequent character of SNS weak link junctions [14], [15] therefore we suppose so far that a very thin residual YBCO film remains in the gap of the SFS structure with the above mentioned critical currents.

After the next FIB etching (current $10 \mathrm{pA}$ and duration $1.5 \mathrm{~min}$ ) we did not observed superconducting properties through the gap, therefore we suppose that YBCO was completely removed but the LRPE was not realized. This confirms the $R-T$ dependence of SFS structure corresponding to typical LSMO dependence (Fig. 5, full line). However, in comparison to as-prepared LSMO $R-$ $T$ dependence (Fig. 5, dashed line) one can see a large $(63 \mathrm{~K})$ shift of the resistance maximum to a lower temperature.

As it was mentioned above the cutting of YBCO film can be accompanied by degradation of the under-laying LSMO film. The decreased temperature of the resistance maximum (transition of LSMO to ferromagnetic state) can be explained by the influence of gallium FIB irradiation on the LSMO.

\section{CONCLUSIONS}

The realization of high quality superconducting weak links or Josephson junctions based on high-Tc superconductors is at present a very difficult task. Some achievements are expected using advanced microcircuit technologies (eg, FIB) for preparation SNS or SFS structures of nanometer dimensions. SFS heterostructures, in comparison with SNS weak links, offer an opportunity for new physical effects (LRPE) as well as new modes of operation in cryoelectronic and cryospintronic circuits (pi-junctions, spin valves). In the paper we present preliminary results on the high-Tc superconducting SFS structure in 
the so-called lateral geometry. In the YBCO/LSMO bilayer the top YBCO layer was disconnected by narrow ( $L \approx 70-80 \mathrm{~nm}$ ) lateral gap in the YBCO microstrip using focused ion beam (FIB) patterning. Results show that FIB offers suitable procedure for realization of nanometer size devices but problems of films degradation by FIB radiation have to be solved. Another separate problem, generation of LRPE in SFS structure, is creation of local magnetic inhomogeneity in the half metal LSMO film. Solution of these problems is the aim of the next period of our investigations.

\section{Acknowledgment}

This publication is the result of the project implementation: CENTE II, ITMS code 26240120019, supported by the Research \& Development Operational Programme funded by the ERDF.

\section{REFERENCES}

[1] BERGERET, F. S.-VOLKOV, A. F.-EFETOV, K. B.: Odd Triplet Superconductivity and Related Phenomena in Superconductor-Ferromagnet Structures, Reviews of Modern Physics $7 \mathbf{7}$ (2005), 1321-1373.

[2] TAO, Y. C.-HU, J. G.: Superconducting Spintronics: SpinPolarized Transport in Superconducting Junctions with Ferromagnetic Semiconducting Contact, Journal of Applied Physics 107 (2010), 041101-041101-13.

[3] ANDREEV, A. F.: Thermal Conductivity of the Intermediate State of Superconductors, Sov. Phys. JETP 19 (1964), $1228-1231$.

[4] BUZDIN, A. I. : Proximity Effects in Superconductor-Ferromagnetic Heterostructures, Reviews of Modern Physics 77 (2005), 935-976.

ESCHRIG, M.-LOFWANDER, T.: Triplet Supercurrents in Clean and Disordered Half-Metallic Ferromagnets, Nature Physics 4 (2008), 138-143.

[5] GOlubOV, A. A.-KUPRIYANOV, M. Yu.-ILICHEV, E. : The Current Phase Relation in Josephson Junctions, Reviews of Modern Physics 77 (2005), 411-469.

[6] HALTERMANN, K.-VALLS, O. T.: Emergence of Triplet Correlations in Superconductor/Half-Metallic Nanojunctions with Spin-Active Interfaces, Physical Review B 80 (2009), 104502-104502-13.

[7] KUPfERSCHMidT, J. N.-BROUWER, P. W.: Enhanced Triplet Andreev Reflection off a Domain Wall in a Lateral Geometry, Physical Review B 80 (2009), 214537-214537-5.

[8] LLOYD, S. J.-MATHUR, N. D.-LOUDON, J. C.-MIDGLEY, P. A.: Magnetic Domain-Wall Width in $\mathrm{La}_{0.7} \mathrm{Ca}_{0.3} \mathrm{MnO}_{3}$ Thin Films Measured using Fresnel Imaging, Physical Review B 64 (2001), 172407-172407-4.

[9] ZIESE, M.-VREJOIU, I.-HESSE, D.: Inverted Hysteresis and Giant Exchange Bias in $\mathrm{La}_{0.7} \mathrm{Sr}_{0.3} \mathrm{MnO}_{3} / \mathrm{SrRuO}_{3}$ Superlattices, Applied Physics Letters 97 (2010), 052504-052504-3.

[10] ŠPANKOVÁ, M.-CHROMIK, Š.-VÁVRA, I.-SEDLÁČKOVÁ, K.-LOBOTKA, P.—LUCAS, S.-STANČEK, S. : Epitaxial LSMO Films Grown on MgO Single Crystalline Substrates, Applied Surface Science 253 (2007), 7599-7603.

[11] TEBANO, A.-ORSini, A.-Di CASTRO, D.-MADAGLIA, O. G.-BALESTRINO, G. : Interplay between Crystallographic Orientation and Electric Transport Properties in $\mathrm{La}_{0.7} \mathrm{Sr}_{0.3} \mathrm{MnO}_{3}$ Films, Applied Physics Letters 96 (2010), 092505-092505-3.
[12] OSZI, Zs.-BENACKA, S.-STRBIK, V.-CHROMIK, S.SPANKOVA, M.-KOSTIC, I.-KLEJA, P.: Properties of $\mathrm{YBa}_{2} \mathrm{Cu}_{3} \mathrm{O}_{x}$ and $\mathrm{Bi}_{2} \mathrm{Sr}_{2} \mathrm{CaCu}_{2} \mathrm{O}_{x}$ Thin Film Microstrips Patterned by Argon Ion Beam, Thin Solid Films 433 (2003), $359-362$.

[13] CHROMIK, Š.-BEŇAČKA, Š.-LEVÁRSKY, J.-PLECENÍK, A.—SCHILDER, J.-ŠMATKO, V.—ŠTRBÍK, V.: High Tc Superconducting Y-Ba-Cu-O Thin Films Prepared by Vacuum Co-Evaporation, Czechoslovak Journal of Physics B 37 (1987), 1317-1320.

14] BENACKA, S.-STRBIK, V.-CHROMIK, S.-ADAM, R.DARULA, M.-GAZI, S. : Mechanisms of Critical Current Limitation in YBCO Thin Film Structures, Fizika Nizkikh Temperatur 24 (1998), 621-623.

[15] DELIN, K. A.-KLEINSASSER, A. W. Stationary Properties of High-Critical-Temperature Proximity Effect Josephson Junctions: Superconductor Science and Technology 9 (1996), $227-269$.

Received 23 October 2010

Vladimír Štrbík (RNDr, CSc) graduated from the Faculty of Natural Sciences of Comenius University in Experimental Physics with specialization on Physics of condensed matter and acoustics in 1977. He started with the Department of Cryoelectronics in the Institute of Electrical Engineering (IEE), Slovak Academy of Sciences (SAS), Bratislava, Slovakia (1977) and he continues to work at IEE SAS up to date. In 1987, he received his PhD. (CSc.) degree in the field of superconducting cryoelectronic devices and circuits. He works at IEE SAS as a scientific worker mainly in the field electrical and magnetic properties of superconducting thin films, structures, devices and circuits. He has solved many domestic and international scientific projects as principal investigator or co-worker. His results have been presented in more than 70 articles in scientific periodicals and more than 50 contributions at international conferences.

Štefan Beňačka (RNDr, CSc), graduated from the Faculty of Natural Sciences of J.A. Comenius University Bratislava (1960) in experimental physics with specialization on physics of high frequencies. He was joined with the Institute of Electrical Engineering, Slovak Academy of Sciences (IEE SAS) from 1960 until 2005. At present he is acting as emeritus professor at the IEE SAS. His research interests are in physics of superconductors, tunneling (Josephson) effects in low and high temperature superconductor weak link junctions, recently, namely interaction of high temperature superconductors (S) with ferromagnetic (F) thin films in SFS weak link junctions of nanometer dimensions.

Štefan Gaži (Mgr), graduated from the Faculty of Natural Sciences of Comenius University Bratislava (1972) in experimental physics with specialization on plasma physics. He started at the same year in the Institute of Electrical Engineering, Slovak Academy of Sciences, focusing his activity in experimental investigation of tunnelling effects in superconducting weak links and Josephson junctions, both in low and high temperature superconductor structures. Recent years he is engaged namely in investigation of superconductor-ferromagnetsuperconductor (SFS) junctions of low temperature superconductors and in the study of arrays of weak links (nanometer dimension) prepared on niobium based thin films and nanocomposites.

Vasilij Šmatko (RNDr) graduated from the Faculty of Physics and Mathematics at the South-Ukrainian University, Odessa, in 1976. He started in the same year in BEZ 
Bratislava. Since 1980 he has worked in the Institute of Electrical Engineering, Slovak Academy of Sciences. At present he is engaged in nanostructures realized by focused ion beam technology and electron microscopy.

Štefan Chromik (Ing, DrSc), graduated from the Faculty of Electrical Engineering at Slovak University of Technology, Bratislava, Slovakia, in 1973. He started with the Department of Cryoelectronics in Institute of Electrical Engineering, Slovak Academy of Sciences (IEE SAS), Bratislava, Slovakia (1973) and he continues to work at IEE SAS up to date. Now he is head of the Department of Cryolectronics. He works at IEE SAS as a scientific worker mainly in the field of preparation of superconducting, ferromagnetic and dielectric thin films. He has solved many domestic and international scientific projects as principal investigator or co-worker. He authored or co-authored more than 110 papers in scientific journals (cited more than 270 times) and 8 patents.

Agáta Dujavová (Mgr, $\mathrm{PhD}$ ) graduated from the Faculty of Natural Sciences at Comenius University in 2006, in the same year she began a PhD study on the Institute of Electrical Engineering Slovak Academy of Sciences (IEE SAS). This year she receives her $\mathrm{PhD}$ in the field of Physical Engineering at IEE SAS. At present she works on high temperature superconducting and ferromagnetic materials research.

Ivo Vávra (Ing, CSc) for biography see page 106 of this issue. 